\title{
Effect of Laparoscopic Adjustable Gastric Banding on Metabolic Syndrome and Its Risk Factors in Morbidly Obese Adolescents
}

\author{
Rushika Conroy, ${ }^{1}$ Eun-Ju Lee, ${ }^{1}$ Amy Jean, ${ }^{1}$ Sharon E. Oberfield, ${ }^{1}$ Aviva Sopher, ${ }^{1}$ \\ Krystina Kiefer, ${ }^{1}$ Courtney Raker, ${ }^{1}$ Donald J. McMahon, ${ }^{1}$ Jeffrey L. Zitsman, ${ }^{2}$ \\ and Ilene Fennoy ${ }^{1}$ \\ ${ }^{1}$ Division of Pediatric Endocrinology, Morgan Stanley Children's Hospital of New York, Columbia University Medical Center, New York, \\ NY 10032, USA \\ ${ }^{2}$ Division of Surgery, College of Physicians and Surgeons, Columbia University Medical Center, New York, NY 10032, USA
}

Correspondence should be addressed to Ilene Fennoy, if1@columbia.edu

Received 18 August 2010; Accepted 6 October 2010

Academic Editor: Francesco Saverio Papadia

Copyright ( $) 2011$ Rushika Conroy et al. This is an open access article distributed under the Creative Commons Attribution License, which permits unrestricted use, distribution, and reproduction in any medium, provided the original work is properly cited.

\begin{abstract}
We examined the effect of laparoscopic adjustable gastric banding (LAGB) on weight loss, inflammatory markers, and components of the Metabolic Syndrome (MeS) in morbidly obese adolescents and determined if those with MeS lose less weight post-LAGB than those without. Data from 14-18 yr adolescents were obtained at baseline, 6 and 12 months following LAGB. Significant weight loss and improvements in MeS components were observed 6 months and one year following LAGB. The incidence of MeS declined $56.8 \%$ after 6 months and $69.6 \%$ after 12 months. There was no significant difference in amount of weight lost post-LAGB between those with and without $\mathrm{MeS}$ at either timepoint. Correlations between change in weight parameters and components of MeS in those with and without MeS at baseline were examined and found to vary by diagnostic category. LAGB is effective for short-term improvement in weight, inflammatory markers, and components of MeS in morbidly obese adolescents.
\end{abstract}

\section{Introduction}

Over the past 30 years, the prevalence of adult obesity in the United States has doubled while that of adolescent obesity has tripled [1]. Current estimates classify $15.5 \%$ of US children and adolescents as overweight (body mass index (BMI) between 85th and 95th percentile for age), 4\% as obese (BMI between 95th and 99th percentile for age), and $4 \%$ as morbidly obese (BMI $\geq 99$ th percentile for age). These children are at risk of developing serious obesityrelated comorbidities such as type 2 diabetes, dyslipidemia, hypertension, and metabolic syndrome (MeS). The necessity for early, aggressive treatment of obesity stems from the need to not only alleviate the medical and psychosocial comorbidities experienced in adolescence but also to decrease the risk of premature mortality in adulthood.

MeS affects an estimated $1 / 3$ to $1 / 2$ of morbidly obese adolescents [2] and likely contributes to the increased morbidity and mortality seen in adulthood. Pediatric MeS, just as adult $\mathrm{MeS}$, is associated with a significantly elevated risk of cardiovascular disease and type 2 diabetes. Additionally, risk of MeS in adulthood is associated with its presence in childhood and adolescence $[3,4]$.

Adolescent obesity has significant long-term consequences, as there is a dose-dependent relationship between BMI in adolescence and risk of morbidity and mortality in adulthood [5]. Alarmingly, studies show that childhood obesity, particularly adolescent obesity, is a key predictor of adult obesity, and up to $77 \%$ of children will carry their obesity into adulthood [6]. The dangers of adult obesity include a reduction of median survival by $8-10$ years in adults with BMIs between 40 and $45 \mathrm{~kg} / \mathrm{m}^{2}$ [7].

Increasing evidence suggests that traditional nonsurgical weight loss methods are ineffective and that bariatric surgery is the most sustainable and effective treatment for weight loss in the morbidly obese [8-11]. Presently, the most common surgical options for adolescents are Roux-en-Y gastric bypass and laparoscopic adjustable gastric banding (LAGB), with 
gastric bypass comprising more than $90 \%$ of US adolescent cases in 2003 [1]. LAGB is associated with a five- to ten-fold lower mortality rate and three-fold lower complication rate than gastric bypass, has the advantages of adjustability and reversibility, and has been associated with sustained weight loss and improved comorbidities in adults [12]. There is little long-term data on LAGB in adolescents however, since the procedure is not FDA approved for use in individuals under the age of 18 yrs.

While a handful of promising national and international studies demonstrating the safety and efficacy of LAGB in morbidly obese adolescents have been published [8-11, 1319], few report on the effects of LAGB-induced weight loss on MeS. The aim of this study was to evaluate the effects of LAGB on weight, inflammatory markers, and components of $\mathrm{MeS}$ in morbidly obese adolescents and to determine if those with $\mathrm{MeS}$ lose less weight post-LAGB than those without as a consequence of their preoperative metabolic derangements.

We hypothesize that LAGB will result in a significant amount of excess weight loss, which will be accompanied by improvements in markers of inflammation as well as in measures diagnostic of MeS. We further postulate that MeS may represent a more advanced state of metabolic derangement, in which the patient is more resistant to weight loss. Therefore, those who carry a diagnosis of $\mathrm{MeS}$ will lose less weight compared to those who do not.

\section{Methods}

This is an IRB-approved, prospective, nonrandomized single center study conducted under an FDA-approved Investigational Device Exemption. An initial feasibility trial of 15 adolescents with followup was performed with interim safety and effectiveness data submitted to the FDA. The FDA subsequently granted approval to implant up to 125 additional patients with evaluation and followup identical to the initial 15. All adolescents who underwent LAGB were entered into a database and included in the data analysis. Subjects with 6-month $(6 \mathrm{~m})$ and/or 12-month $(12 \mathrm{~m})$ post-LAGB anthropometric and/or metabolic data were included in this report, which assessed weight, BMI, waist circumference (WC), systolic and diastolic blood pressure (BP) percentiles, triglycerides (TG), high-density lipoprotein (HDL), fasting blood sugar (FBS), C-reactive protein (CRP), $\%$ weight loss $(\% \mathrm{WL})$, and $\%$ excess weight loss (\%EWL). Inclusion and exclusion criteria are described in Table 1. The BMI guidelines used were consistent with NIH criteria for bariatric surgery in adults [20].

2.1. Presurgical Methods. Subjects were recruited from among those enrolled in weight management programs at Columbia University Medical Center (CUMC) or referred by private pediatricians to the Center for Adolescent Bariatric Surgery. Consent was obtained from parents or caregivers and assent obtained from each subject. Both adolescents and their parents or caregivers were actively involved in the screening process, during which medical, social, psychiatric, and weight histories were obtained. As part of the protocol, subjects who met criteria for entry were enrolled at CUMC in either a $6 \mathrm{~m}$ lifestyle modification program if they had no prior programmatic weight loss experience or a $3 \mathrm{~m}$ program if they had documentation of participation in prior programs. During this time, subjects met monthly with a nurse practitioner/exercise specialist and a registered dietician, one to three times with an endocrinologist, and twice with a psychiatrist or psychologist and the pediatric surgeon. At the end of the lifestyle modification program, those subjects who demonstrated active participation and willingness to make changes were offered LAGB, while those having difficulty with program compliance were encouraged to continue with medical weight management. Prior to undergoing LAGB, baseline evaluations including anthropometric measurements, fasting blood studies (comprehensive metabolic panel, lipids, and nutrition panel), and an oral glucose tolerance test were performed. In addition, an electrocardiogram, chest X-ray, sleep study, and abdominal ultrasound with attention to the liver and gallbladder were completed for preoperative clearance. A bone age was performed to document skeletal maturity, and a serum HCG was carried out in females to ensure negative pregnancy.

Comorbidities were diagnosed by medical staff using the following criteria. Diagnosis of MeS was made using the 2003 Cook criteria for adolescents with modification of the FBS criterion to the November 2003 ADA criterion of $>100 \mathrm{mg} / \mathrm{dl}$ as abnormal. Thus, we used the presence of any 3 of the following: TG $\geq 110 \mathrm{mg} / \mathrm{dL}, \mathrm{HDL} \leq 40 \mathrm{mg} / \mathrm{dL}, \mathrm{FBS} \geq$ $100 \mathrm{mg} / \mathrm{dL}$, WC $(\mathrm{cm}) \geq$ 90th percentile for ethnicity, age and sex, and BP $(\mathrm{mmHg}) \geq 90$ th percentile for age, height, and sex [21]. Hypertension was defined as systolic and/or diastolic BP greater than the 95th percentile for age, sex, and height [22]. Dyslipidemia was defined by the presence of fasting HDL $\leq 40 \mathrm{mg} / \mathrm{dl}$, LDL $\geq 110 \mathrm{mg} / \mathrm{dl}$, TG $\geq$ $110 \mathrm{mg} / \mathrm{dl}$, and/or total cholesterol $\geq 200 \mathrm{mg} / \mathrm{dl}$.

Height was measured using a stadiometer to the nearest $0.1 \mathrm{~cm}$; weight was measured using a digital readout scale to the nearest $0.1 \mathrm{~kg}$. WC was measured at the anterior superior iliac spine to the nearest $0.5 \mathrm{~cm}$. BP was measured with an aneroid sphygmomanometer while subjects were seated. Three readings for each of systolic and diastolic BPs were obtained, and the average of the measurements was used. Blood samples were obtained after an overnight fast. Serum glucose, HDL, and TG were run on an Olympus A42700 analyzer (Olympus America Inc, Melville NY). CRP was measured by nephelometry using a BN2 immunoassay (Siemens Industry, USA).

2.2. Surgical Methods. LAP-BAND (LAP-BAND System; Allergan Corp, Santa Barbara, CA) is a silicone ring with an adjustable inner diameter that is positioned around the proximal stomach just distal to the gastroesophageal junction, creating a small proximal gastric pouch. Kinkresistant tubing connects the the band to a subcutaneous access port. Saline can be injected into or withdrawn from the port to adjust the diameter of the band. LAP-BAND controls the outlet diameter of the upper stomach pouch, limiting emptying rate and causing a feeling of early satiety and subsequent weight loss. 
All surgical procedures were performed at the Morgan Stanley Children's Hospital of New York by a pediatric surgeon (JZ). Placement of the LAP-BAND was performed laparoscopically with the patient under general anesthesia. The LAP-BAND was placed using 5 trocar sites according to the pars flaccida technique, which has been described in detail elsewhere [23]. The LAP-BAND was left empty at the end of placement to allow for postoperative swelling. Patients were observed in the hospital overnight, and a contrast esophagram was performed to confirm band position and assess pouch emptying prior to discharge.

2.3. Follow-Up Assessment. Patients were instructed to return for followup visits for assessment of weight changes, for nutritional advice, and for postsurgical monitoring and adjustments at weeks $2,4,6$, and 8 , then monthly for the initial $12 \mathrm{~m}$, with plans for followup at 15, 18, and $24 \mathrm{~m}$, and semiannually thereafter for a total of 5 years. Fasting laboratory evaluations (including comprehensive metabolic panel, nutritional panel, lipids, and oral glucose tolerance test) were performed at postoperative months 6 and 12 with biannual assessments intended for the next 5 yrs. The first band adjustment occurred 5-6 weeks postLAGB. Subsequent band adjustments were tailored to the individual's needs, including feelings of hunger and satiety, as well as pain with or without vomiting. The overall goal for weight loss was $1-1.5 \mathrm{lbs}$ per week, which is consistent with other studies $[16,24]$. Patients were instructed to follow a pureed diet in the first postoperative week, a blended diet for postoperative weeks $2-3$, a soft diet for postoperative weeks 4-6, and a well-balanced low-fat diet for postoperative week 7 and beyond. In addition, patients were instructed to continue physical activity, to eat 3 small meals per day, to avoid liquids 30 minutes before and 30 minutes after eating, and to stop eating when full.

Ideal body weight was derived by multiplying the square of each subject's baseline height by the BMI at the 85th percentile for each subject's sex and age using the CDC growth charts (2000). There is no consensus with respect to the optimal BMI to use when determining ideal body weight for children and adolescents. Our goal was to have subjects achieve a weight and BMI within normal range for their age. While a BMI at the 85th percentile is at the uppermost limit of normal, it is within the normal range and a realistic goal for our study subjects. Excess weight (EW) was calculated by subtracting ideal body weight from actual baseline weight. $\% \mathrm{EWL}$ was calculated by dividing the amount of weight lost at $6 \mathrm{~m}$ or $12 \mathrm{~m}$ post-LAGB by the EW and multiplying by 100 . $\% \mathrm{WL}$ was calculated by dividing the amount of weight lost at $6 \mathrm{~m}$ or $12 \mathrm{~m}$ post-LAGB by baseline weight and multiplying by 100 .

2.4. Statistical Analysis. Pearson correlation was used to identify associations among changes in weight and indices of the MeS at both $6 \mathrm{~m}$ and $12 \mathrm{~m}$. Fisher's exact test was used to assess the change in the proportion of subjects meeting MeS criterion at $6 \mathrm{~m}$ and $12 \mathrm{~m}$. Logistic regression with dichotomously coded MeS status (improved versus

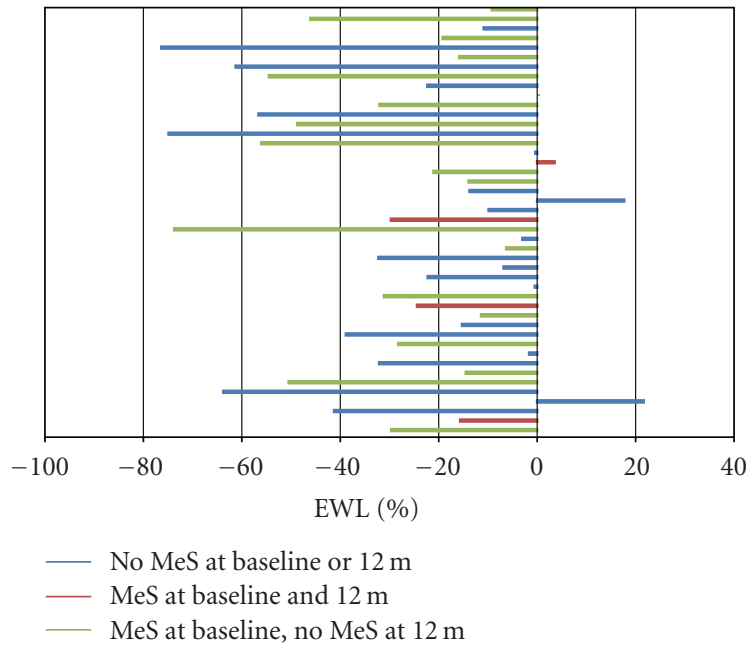

FIgURE 1: \%EWL $12 \mathrm{~m}$ post-LAGB.

unimproved) was used to assess which weight change measure was most highly associated with change in MeS status. Linear mixed models for repeated measures were used to check whether average within-subject changes over time represented statistically reliable changes from baseline. The $6 \mathrm{~m}$ and $12 \mathrm{~m}$ cohorts were analyzed separately. A $P$-value less than. 05 was considered statistically significant, and data are expressed as counts and percentages or means and standard error of the mean (SEM). No adjustments for analysis of multiple outcome measures were made.

\section{Results}

All procedures were performed laparoscopically. There were no operative deaths. Early complications included 1 exploration for bleeding, 1 repositioning of a misplaced band, and 1 exploration for presumed bowel obstruction which proved to be prolonged ileus. One patient developed aggravation of plantar fasciitis requiring analgesics and physical therapy. There were 2 minor wound complications. Late complications included 3 band displacements which required laparoscopic repositioning and 5 port repositions. No patient required band removal.

From March 2006 through June 2010, 108 adolescents ( 31 males, 77 females) underwent LAGB. At the time of this report, all subjects were at least $6 \mathrm{~m}$ post-LAGB and $88(81.5 \%)$ presented for followup. These 88 adolescents (36 Hispanic, 13 African-American, 33 Caucasian, and 6 mixed ethnicity) had baseline age $16.8 \pm 0.1 \mathrm{yrs}$, BMI $45.9 \pm$ $1.0 \mathrm{~kg} / \mathrm{m}^{2}$, and EW $66.9 \pm 3.0 \mathrm{~kg}$. Thirty-seven subjects had MeS. Mean \%WL at $6 \mathrm{~m}$ post-LAGB was $7.5 \pm 0.9 \%$, ranging from a loss of $24.8 \%$ to a gain of $12.7 \%$. The mean $\% \mathrm{EWL}$ equaled $17.1 \pm 2.2 \%$, ranging from $86.8 \%$ of excess weight lost to $27.3 \%$ of excess weight gained. Fifteen patients gained weight at the $6 \mathrm{~m}$ time point with mean weight gain of $6.1 \pm 1.1 \mathrm{~kg}$.

Sixty-two subjects (57\%) provided blood work for evaluation. Baseline and $6 \mathrm{~m}$ post-LAGB anthropometric 
TABle 1: Patient selection criteria.

Inclusion Criteria

- 14-18 yrs old at time of enrollment

- BMI $\geq 40 \mathrm{~kg} / \mathrm{m}^{2}$ or $\geq 35 \mathrm{~kg} / \mathrm{m}^{2}$ and at least 1 comorbidity

- Obesity for at least 5 yrs with documented attempts at diet and medical management

- Bone age at least $13.5(\mathrm{~F})$ and $14.5(\mathrm{M})$

- Emotional maturity

- Use of appropriate contraception (F)

- Understanding and willingness to comply with protocol

(F): female; (M): male; NSAID: non-steroidal anti-inflammatory drug.
Exclusion Criteria

- History of prior bariatric surgery or intent to have additional bariatric surgery in the next year

- History of GI tract anomalies, severe cardiopulmonary disease, coagulopathy, hepatic insufficiency or cirrhosis

- History of gastric or esophageal surgery

- History of chronic aspirin and/or NSAID use

- Pregnancy or intent to become pregnant in the next year

- Eating disorders with self-induced vomiting

- Inability to understand the intervention and followup

TABLE 2: Clinical characteristics and MeS variables at baseline and at $6 \mathrm{~m}$ post-LAGB $(N=62)$.

\begin{tabular}{lccc}
\hline Variable & Timepoint & & \\
& Baseline & 6 m post-LAGB & $\mathrm{P}^{\dagger}$ \\
\hline BMI $\left(\mathrm{kg} / \mathrm{m}^{2}\right)$ & $47.8 \pm 0.95(35.7-86.2)$ & $44.0 \pm 1.0(28.6-81.9)$ & $<.0001$ \\
Weight $(\mathrm{kg})$ & $134.9 \pm 3.6(83.9-201.7)$ & $125.5 \pm 3.6(69.4-195.5)$ & $<.0001$ \\
WC $(\mathrm{cm})$ & $136.1 \pm 2.4^{*}(107.5-188)$ & $127.8 \pm 2.4(91.8-177.5)$ & $<.0001$ \\
Systolic BP (\%ile) & $70.9 \pm 3.3^{\#}(6-100)$ & $59.6 \pm 3.3(5-99)$ & .0056 \\
Diastolic BP (\%ile) & $74.5 \pm 2.8^{\#}(23-100)$ & $62.3 \pm 2.8(6-99)$ & .0015 \\
TG (mg/dl) & $1.30 \pm 0.10(0.44-5.57)$ & $1.18 \pm 0.10(0.32-4.27)$ & .065 \\
FBS (mg/dl) & $4.62 \pm 0.06(3.39-6.44)$ & $4.70 \pm 0.06(3.77-5.77)$ & $\mathrm{NS}$ \\
HDL (mg/dl) & $1.09 \pm 0.03(0.75-1.81)$ & $1.12 \pm 0.03(0.75-1.68)$ & $\mathrm{NS}$ \\
CRP $(\mathrm{mg} / \mathrm{L})$ & $93.3 \pm 10.5^{*}(2.29-440.0)$ & $66.7 \pm 10.5(3.05-363.8)$ & .0002 \\
\hline
\end{tabular}

Data are presented as Mean \pm SEM (Range). ${ }^{\dagger} P$-value for changes in variables between baseline and $6 \mathrm{~m}$ post-LAGB. ${ }^{*} N: 59 .{ }^{\#} N: 60$.

Table 3: Clinical characteristics and MeS variables at baseline and $12 \mathrm{~m}$ post-LAGB $(N=29)$.

\begin{tabular}{|c|c|c|c|}
\hline \multirow{2}{*}{ Variable } & \multicolumn{3}{|c|}{ Timepoint } \\
\hline & Baseline & $12 \mathrm{~m}$ post-LAGB & $P^{\dagger}$ \\
\hline $\operatorname{BMI}\left(\mathrm{kg} / \mathrm{m}^{2}\right)$ & $48.8 \pm 1.4(35.9-65.4)$ & $42.9 \pm 1.5(27.7-65.8)$ & $<.0001$ \\
\hline Weight (kg) & $135.0 \pm 5.2(94.5-198.4)$ & $120.2 \pm 5.2(68.4-167.6)$ & $<.0001$ \\
\hline $\mathrm{WC}(\mathrm{cm})$ & $135.4 \pm 3.6^{*}(109.5-168)$ & $123.5 \pm 3.5(87-165)$ & $<.0001$ \\
\hline SBP (\%-ile) & $71.1 \pm 5.0(6-98)$ & $58.1 \pm 5.1(5-100)$ & .019 \\
\hline DBP (\%-ile) & $71.7 \pm 3.9(25-100)$ & $68.0 \pm 4.0(16-98)$ & NS \\
\hline TG (mg/dl) & $1.30 \pm 0.13(4.97-3.15)$ & $1.22 \pm 0.13^{\#}(4.07-4.38)$ & NS \\
\hline FBS (mg/dl) & $4.80 \pm 0.11(3.94-7.44)$ & $4.66 \pm 0.11(4.05-6.16)$ & NS \\
\hline HDL (mg/dl) & $1.10 \pm 0.05(0.78-1.66)$ & $1.19 \pm 0.05^{\#}(0.73-1.68)$ & .029 \\
\hline CRP (mg/L) & $77.1 \pm 11.4^{*}(7.0-257.1)$ & $53.3 \pm 11.4^{\#}(2.1-221.9)$ & .024 \\
\hline
\end{tabular}

Data are presented as Mean \pm SEM (Range). ${ }^{\dagger} P$-value for changes in variables between baseline and $12 \mathrm{~m}$ post-LAGB. ${ }^{*} N: 27 .{ }^{\#} N: 28$.

TABle 4: $P$-values for correlations among weight change measures and components of MeS at $6 \mathrm{~m}$ post-LAGB by MeS status at baseline.

\begin{tabular}{|c|c|c|c|c|c|c|}
\hline \multirow{2}{*}{ Variable } & \multicolumn{3}{|c|}{ No $\operatorname{MeS}(N=51)$} & \multicolumn{3}{|c|}{ Yes $\operatorname{MeS}(N=37)$} \\
\hline & \%EWL & $\% \mathrm{WL}$ & BMI & $\%$ EWL & $\% \mathrm{WL}$ & BMI \\
\hline $\mathrm{WC}(\mathrm{cm})$ & $<0.0001$ & 0.01 & $<0.0001$ & 0.0024 & 0.0004 & 0.0005 \\
\hline $\mathrm{HDL}(\mathrm{mg} / \mathrm{dl})$ & NS & NS & NS & NS & NS & NS \\
\hline TG (mg/dl) & NS & 0.0098 & 0.004 & NS & NS & NS \\
\hline CRP (mg/dl) & NS & NS & NS & NS & NS & NS \\
\hline FBS (mg/dl) & NS & 0.019 & 0.013 & NS & NS & NS \\
\hline SBP \%ile & NS & NS & 0.06 & NS & NS & NS \\
\hline DBP \%ile & NS & NS & NS & NS & NS & NS \\
\hline
\end{tabular}


TABLE 5: $P$-values for correlations among weight change measures and components of MeS at $12 \mathrm{~m}$ post-LAGB by MeS status at baseline.

\begin{tabular}{|c|c|c|c|c|c|c|}
\hline \multirow{2}{*}{ Variable } & \multicolumn{3}{|c|}{ No $\operatorname{MeS}(N=22)$} & \multicolumn{3}{|c|}{ Yes $\operatorname{MeS}(N=23)$} \\
\hline & $\%$ EWL & $\% \mathrm{WL}$ & BMI & $\%$ EWL & $\% \mathrm{WL}$ & BMI \\
\hline $\mathrm{WC}(\mathrm{cm})$ & $<0.0001$ & $<0.0001$ & $<0.0001$ & 0.043 & 0.009 & 0.002 \\
\hline $\mathrm{HDL}(\mathrm{mg} / \mathrm{dl})$ & NS & NS & NS & 0.032 & 0.047 & 0.08 \\
\hline $\mathrm{TG}(\mathrm{mg} / \mathrm{dl})$ & NS & NS & NS & NS & NS & NS \\
\hline CRP (mg/dl) & NS & NS & NS & NS & NS & NS \\
\hline FBS (mg/dl) & NS & 0.046 & 0.037 & NS & NS & NS \\
\hline SBP \%ile & NS & NS & NS & NS & NS & NS \\
\hline DBP \%ile & 0.067 & NS & 0.065 & NS & NS & NS \\
\hline
\end{tabular}

data of subjects who had blood work assessed were not significantly different from that of subjects who did not (data not shown). There were significant improvements in BMI, weight, WC, systolic and diastolic BP\% iles, and CRP (Table 2). MeS resolved in 21 of the adolescents diagnosed at baseline $(56.8 \% ; P<.0004)$. No significant differences were observed in \%EWL, \%WL, or change in BMI between those with $\mathrm{MeS}$ at baseline and those without (data not shown). Resolution of MeS did not significantly correlate with change in BMI, \%WL, or \%EWL ( $P=\mathrm{NS})$.

At the time of this report, 84 adolescents were at least $12 \mathrm{~m}$ post-LAGB. Forty-five adolescents (50.6\%; 17 male, 28 female) attended $12 \mathrm{~m}$ followup visits. These 45 adolescents (19 Hispanic, 6 African-American, 17 Caucasian, and 3 mixed ethnicity) had baseline age $17.2 \pm 0.2$ yrs, BMI $45.8 \pm$ $1.5 \mathrm{~kg} / \mathrm{m}^{2}$, and EW $69.4 \pm 4.3 \mathrm{~kg}$. Twenty-three subjects had $\mathrm{MeS}$. The $\% \mathrm{WL}$ for this group was $10.9 \pm 1.2 \%$, ranging from a $43.0 \%$ loss to a gain of $9.1 \%$. Mean $\%$ EWL was $25.9 \pm 3.6 \%$ ranging from an excess weight loss of $76.4 \%$ to an excess weight gain of $21.6 \%$ (Figure 1). Four adolescents had net weight gains, the mean of which was $6.1 \pm 2.8 \mathrm{~kg}$ (range $0.6-$ $13.5 \mathrm{~kg})$.

Twenty nine (35\%) provided bloodwork for evaluation. There were no differences in the anthropometric data between those who came for bloodwork and those who did not (data not shown). Significant improvements in BMI, weight, WC, systolic BP \%ile, CRP, and HDL one year after LAGB were observed (Table 3). MeS resolved in 16 of the adolescents diagnosed at baseline (69.6\%; $P=.0003)$. There were no significant differences in $\% \mathrm{EWL}, \% \mathrm{WL}$, or change in BMI between those who had MeS at baseline and those who did not (data not shown). Resolution of MeS correlated to $\%$ EWL, nearing statistical significance $(P=.06)$ but did not correlate to \%WL or BMI ( $P=\mathrm{NS})$.

Tables 4 and 5 demonstrate correlations of components of MeS with parameters of weight change in those with and without MeS at $6 \mathrm{~m}$ and $12 \mathrm{~m}$ post-LAGB. At $6 \mathrm{~m}$ post-LAGB, \%WL, BMI, and \%EWL (Table 4) correlated significantly with WC in those who did not have MeS at baseline. BMI and \%WL also correlated significantly with change in TG and FBS in this cohort. In those with MeS at baseline, BMI, \%EWL, and \%WL only correlated with change in WC (Table 4). One year post-LAGB, \%EWL, BMI, and \%WL correlated significantly with WC in those who did not have MeS at baseline (Table 5). \%WL and BMI, correlated significantly with change in FBS as well. In those with $\mathrm{MeS}$ at baseline, \%EWL, BMI and \%WL correlated with WC and HDL (Table 5).

\section{Discussion}

This study examined the effects of LAGB on a diverse population of morbidly obese adolescents with respect to changes in weight and resolution of MeS. Our results indicate that LAGB is effective in most morbidly obese adolescents for achieving weight loss and decreasing the frequency of MeS. Similar to other adolescent LAGB series, there were no intraoperative or postoperative deaths, and the complication rate was comparable to what has previously been reported [8-10, 14-19]. Our study showed weight loss at $6 \mathrm{~m}$ and $12 \mathrm{~m}$ post-LAGB, which is consistent with results of other studies, but of smaller magnitude. Post-LAGB weight gain was observed in 15 of 88 adolescents at $6 \mathrm{~m}$ post-LAGB and in 4 of 45 adolescents at $12 \mathrm{~m}$ post-LAGB. While Widhalm et al. also reported weight gain in 4 of 7 adolescents post-LAGB, they began gaining at $44 \mathrm{~m}$ after surgery [17]. Although they did not report adolescents who gained weight postoperatively, Silberhumer et al. and Angrisani et al. did describe failure rates of 3 out of 50 at 1-year followup and 5 out of 25 at 5-year followup, respectively, defining failure as $\%$ EWL $<25 \%[13,14]$.

Possible explanations for the insufficient weight loss and weight gain in our subjects include lack of social support, failure to change eating habits, and failure to incorporate recommended exercise. Degree of adherence to followup appointments could further contribute to disappointing outcomes. Possible reasons for lack of followup may include difficulty in attending appointments due to school, work, or other obligations; inability to finance appointments due to loss of health insurance; satisfaction with weight loss such that no need was felt to see the medical team; embarrassment at the lack of weight loss or presence of weight gain; lack of desire to undergo blood testing and/or consume glucola for the 2-hour oral glucose tolerance test. We were unable to provide monetary or other incentives for attending followup visits and complying with lifestyle modifications. Such enticements may have increased our compliance rate and resulted in even better weight loss results. Information regarding changes in body composition, 
including percent fat mass, was not obtained during the study. It is possible that for some adolescents muscle accrual is occurring but not being accounted for when they step on the scale. We plan to assess body fat percentage of our study subjects in future visits in order to better understand body compositional changes that are occurring with weight changes. We are also investigating factors relating to subject compliance in order to understand which adolescents will succeed post-LAGB, thus enabling us to better screen future candidates.

There is increasing evidence that resolution of obesityrelated comorbidities such as $\mathrm{MeS}$ with weight loss can be seen in adolescents as it has been in adults [16, 19, 25]. Holterman et al., using the International Diabetes Federation guidelines, reported a $34 \%$ reduction of $\mathrm{MeS}$ in 10 adolescent girls at $6 \mathrm{~m}$ and a $68 \%$ reduction at $9 \mathrm{~m}$ post-LAGB [16]. Our data show comparable post-LAGB improvement with a $56.8 \%$ resolution of MeS $6 \mathrm{~m}$ postLAGB and a $69.6 \%$ resolution of MeS $12 \mathrm{~m}$ post-LAGB. Both series noted near statistically significant improvement in TG $6 \mathrm{~m}$ post-LAGB, but this was not sustained at the $12 \mathrm{~m}$ time point in our subjects. This is likely a reflection of dietary changes, which incorporated more carbohydrate-rich foods and fewer fatty foods. Consistent with Holterman et al., who saw improvements in systolic BP following LAGB, we saw significant improvement in systolic BP $6 \mathrm{~m}$ and $12 \mathrm{~m}$ post-LAGB. In addition, we saw significant improvement in diastolic BP at the $6 \mathrm{~m}$ time point that was not sustained $12 \mathrm{~m}$ after surgery. Change in HDL approached statistical significance $6 \mathrm{~m}$ post-LAGB and was significant one year following surgery, probably a reflection of the increase in physical activity in our subjects over time.

Studies indicate that $10 \% \mathrm{WL}$ can lead to resolution of a number of adiposity-related comorbidities [26]. In our study, subjects lost only 7.5\% WL $6 \mathrm{~m}$ following surgery, but this gave them a \%EWL of $17.1 \%$. This degree of EWL was accompanied by a significant resolution of MeS in these subjects, manifested by significant improvement in a number of components that define the disease. One year following surgery, with \%WL of $10.9 \%$ and \%EWL of $25.9 \%$, an even greater percentage of subjects had resolution of MeS. It is possible therefore that lesser degrees of WL than the generally recommended $10 \%$ in the morbidly obese population will result in significant health-related improvement and that the utilization of \%EWL to assess co-morbidity risk reduction may be of benefit in the morbidly obese population.

Similar to the observations of Widhalm et al., we also observed improvements in CRP, an inflammatory biomarker that independently predicts future vascular events [19]. Decreased CRP has been associated with reductions in the occurrence of adverse cardiovascular outcomes [27]. In our adolescents, CRP decreased significantly at $6 \mathrm{~m}$ postLAGB and continued to decrease at $12 \mathrm{~m}$ post-LAGB. This improvement is important since $20.5 \%$ of our subjects had at least three risk factors for cardiovascular disease at baseline, which included obesity, sedentary lifestyle, dyslipidemia, elevated BP, impaired glucose tolerance, and impaired FBS. LAGB-induced weight loss appears to eliminate some of these risk factors, in addition to reducing CRP levels, all of which may lead to improved cardiovascular health in this group of adolescents.

This is one of the first studies to date to examine correlations of MeS components to changes in weight in adolescents following bariatric surgery. At both $6 \mathrm{~m}$ and $12 \mathrm{~m}$ post-LAGB, change in WC was correlated with $\% \mathrm{EWL}$, $\% \mathrm{WL}$, and change in BMI, irrespective of MeS status. This is similar to findings by Coppen et al., who observed significant correlation between weight change and WC following a $75.5 \%$ decrease in MeS following 10 weeks of diet-induced weight loss in a group of 6-19 year old [28]. No relationships were observed with any other metabolic parameter of MeS in this study. Our results showed that in those without MeS at baseline, \%WL and change in BMI correlated with change in FBS and TG $6 \mathrm{~m}$ post-LAGB, findings that were not observed in those who had MeS prior to surgery. Despite weight loss and improvements in individual components of MeS, no significant relationship existed between these components and weight loss in those with MeS prior to surgery. This suggests that another factor may serve as a link between weight change and MeS components and is relevant only in those who have the syndrome.

$12 \mathrm{~m}$ following LAGB, \%WL and change in BMI continued to correlate with FBS but not with TG in those who did not have MeS at baseline. The fact that there was no significant improvement in TG levels $12 \mathrm{~m}$ postLAGB could explain this finding. Interestingly, in those who did have MeS at baseline, change in HDL correlated significantly with \%EWL and \%WL. It is as yet unclear what the significance and implications of these findings are. Further studies examining the relationship between MeS components and weight change will help shed more light into this matter.

This study is limited by the lack of a control group undergoing conventional diet and exercise, as was present in a study by O'Brien et al. [8]. The short-term nature of our study precludes an assessment of the long-term impact of LAGB on comorbidities; however, long-term followup is ongoing with a goal of a total of 5 years of postsurgical followup. Our study is not unique in its difficulty with ensuring followup [24]. The number of subjects who presented for followup metabolic evaluation was less than the total number of subjects who presented for followup anthropometric assessment. There were no significant differences in weight change between those who had laboratory data for evaluation and those who did not, suggesting that our conclusions from the laboratory assessments may be extended to the entire group at each time point.

Our study adds to the limited literature documenting the effectiveness of LAGB in achieving not only weight loss with limited adverse effects, but also resolution of comorbidities, particularly MeS in morbidly obese adolescents. We found that $\mathrm{MeS}$ does not hinder weight loss in our study subjects and that a number of components of MeS improve with less than the \%WL currently recommended for improvement. The option of using \%EWL as a goal for improvement of comorbidities in the morbidly obese and obese population is introduced; further studies into this matter are warranted. 
We show that correlations between parameters of weight change and components of MeS indeed are different between those with and without MeS prior to undergoing surgical weight loss. Future research into the implications of these relationships is necessary. Only continued long-term followup can clarify the role of LAGB amongst the various weight loss treatments available and whether the diagnosis of $\mathrm{MeS}$ alters the response of the individual components of $\mathrm{MeS}$ to weight loss.

\section{Acknowledgment}

Each author has reviewed and approved the current version of the manuscript, accepts full responsibility for it and acknowledges that there are no conflicts of interest. No honoraria, grant, or other form of payment was given to anyone to produce the manuscript.

\section{References}

[1] S. A. Xanthakos, "Bariatric surgery for extreme adolescent obesity: indications, outcomes, and physiologic effects on the gut-brain axis," Pathophysiology, vol. 15, no. 2, pp. 135-146, 2008.

[2] T. H. Inge, S. A. Xanthakos, and M. H. Zeller, "Bariatric surgery for pediatric extreme obesity: now or later?" International Journal of Obesity, vol. 31, no. 1, pp. 1-14, 2007.

[3] J. A. Morrison, L. A. Friedman, P. Wang, and C. J. Glueck, "Metabolic syndrome in childhood predicts adult metabolic syndrome and type 2 diabetes mellitus 25 to 30 years later," Journal of Pediatrics, vol. 152, no. 2, pp. 201-206, 2008.

[4] J. A. Morrison, L. A. Friedman, and C. Gray-McGuire, "Metabolic syndrome in childhood predicts adult cardiovascular disease 25 years later: the Princeton lipid research clinics follow-up study," Pediatrics, vol. 120, no. 2, pp. 340-345, 2007.

[5] D. S. Freedman, H. S. Kahn, Z. Mei et al., "Relation of body mass index and waist-to-height ratio to cardiovascular disease risk factors in children and adolescents: the Bogalusa Heart Study," American Journal of Clinical Nutrition, vol. 86, no. 1, pp. 33-40, 2007.

[6] D. S. Freedman, Z. Mei, S. R. Srinivasan, G. S. Berenson, and W. H. Dietz, "Cardiovascular risk factors and excess adiposity among overweight children and adolescents: the Bogalusa heart study," Journal of Pediatrics, vol. 150, no. 1, pp. 12-17, 2007.

[7] G. Whitlock, S. Lewington, P. Sherliker et al., "Body-mass index and cause-specific mortality in 900000 adults: collaborative analyses of 57 prospective studies," Lancet, vol. 373, no. 9669, pp. 1083-1096, 2009.

[8] P. E. O’Brien, S. M. Sawyer, C. Laurie et al., "Laparoscopic adjustable gastric banding in severely obese adolescents: a randomized trial," Journal of the American Medical Association, vol. 303, no. 6, pp. 519-526, 2010.

[9] K. Dolan, L. Creighton, G. Hopkins, and G. Fielding, "Laparoscopic gastric banding in morbidly obese adolescents," Obesity Surgery, vol. 13, no. 1, pp. 101-104, 2003.

[10] B. E. Dillard III, V. Gorodner, C. Galvani et al., "Initial experience with the adjustable gastric band in morbidly obese US adolescents and recommendations for further investigation," Journal of Pediatric Gastroenterology and Nutrition, vol. 45, no. 2, pp. 240-246, 2007.
[11] E. P. Nadler, H. A. Youn, C. J. Ren, and G. A. Fielding, "An update on 73 US obese pediatric patients treated with laparoscopic adjustable gastric banding: comorbidity resolution and compliance data," Journal of Pediatric Surgery, vol. 43, no. 1, pp. 141-146, 2008.

[12] P. E. O’Brien, J. B. Dixon, C. Laurie et al., "Treatment of mild to moderate obesity with laparoscopic adjustable gastric banding or an intensive medical program: a randomized trial," Annals of Internal Medicine, vol. 144, no. 9, pp. 625-633, 2006.

[13] G. R. Silberhumer, K. Miller, S. Kriwanek, K. Widhalm, A. Pump, and G. Prager, "Laparoscopic adjustable gastric banding in adolescents: the Austrian experience," Obesity Surgery, vol. 16, no. 8, pp. 1062-1067, 2006.

[14] L. Angrisani, F. Favretti, F. Furbetta et al., "Obese teenagers treated by Lap-Band System: the Italian experience," Surgery, vol. 138, no. 5, pp. 877-881, 2005.

[15] S. Horgan, M. J. Holterman, G. R. Jacobsen et al., "Laparoscopic adjustable gastric banding for the treatment of adolescent morbid obesity in the United States: a safe alternative to gastric bypass," Journal of Pediatric Surgery, vol. 40, no. 1, pp. 86-91, 2005.

[16] A.-X. Holterman, A. Browne, B. E. Dillard III et al., "Shortterm outcome in the first 10 morbidly obese adolescent patients in the FDA-approved trial for laparoscopic adjustable gastric banding," Journal of Pediatric Gastroenterology and Nutrition, vol. 45, no. 4, pp. 465-473, 2007.

[17] K. Widhalm, S. Dietrich, and G. Prager, "Adjustable gastric banding surgery in morbidly obese adolescents: experiences with eight patients," International Journal of Obesity, vol. 28, supplement 3, pp. S42-S45, 2004.

[18] S. Abu-Abeid, N. Gavert, J. M. Klausner, and A. Szold, "Bariatric surgery in adolescence," Journal of Pediatric Surgery, vol. 38, no. 9, pp. 1379-1382, 2003.

[19] A. R. Al-Qahtani, "Laparoscopic adjustable gastric banding in adolescent: safety and efficacy," Journal of Pediatric Surgery, vol. 42, no. 5, pp. 894-897, 2007.

[20] Consensus Development Conference, "Gastrointestinal surgery for severe obesity," Annals of Internal Medicine, vol. 115, no. 12, pp. 956-961, 1991.

[21] S. Cook, M. Weitzman, P. Auinger, M. Nguyen, and W. H. Dietz, "Prevalence of a metabolic syndrome phenotype in adolescents: findings from the Third National Health and Nutrition Examination Survey, 1988-1994," Archives of Pediatrics and Adolescent Medicine, vol. 157, no. 8, pp. 821827, 2003.

[22] T. Reinehr, M. Kleber, and A. M. Toschke, "Lifestyle intervention in obese children is associated with a decrease of the metabolic syndrome prevalence," Atherosclerosis, vol. 207, no. 1, pp. 174-180, 2009.

[23] C. J. Ren and G. A. Fielding, "Laparoscopic adjustable gastric banding: surgical technique," Journal of Laparoendoscopic and Advanced Surgical Techniques A, vol. 13, no. 4, pp. 257-263, 2003.

[24] F. Favretti, P. E. O'Brien, and J. B. Dixon, "Patient management after LAP-BAND placement," American Journal of Surgery, vol. 184, no. 6, pp. S38-S41, 2002.

[25] C. Gazzaruso, S. Giordanetti, A. La Manna et al., "Weight loss after Swedish adjustable gastric banding: relationships to insulin resistance and metabolic syndrome," Obesity Surgery, vol. 12, no. 6, pp. 841-845, 2002.

[26] X. P. Pi-Sunyer et al., "Clinical guidelines on the identification, evaluation, and treatment of overweight and obesity in adults," NIH Publication, vol. 98, no. 4083, pp. 13-292, 1998. 
[27] P. M. Ridker, E. Danielson, F. A. H. Fonseca et al., "Rosuvastatin to prevent vascular events in men and women with elevated C-reactive protein," New England Journal of Medicine, vol. 359, no. 21, pp. 2195-2207, 2008.

[28] A. M. Coppen, J. A. Risser, and P. D. Vash, "Metabolic syndrome resolution in children and adolescents after 10 weeks of weight loss," Journal of the Cardiometabolic Syndrome, vol. 3, no. 4, pp. 205-210, 2008. 


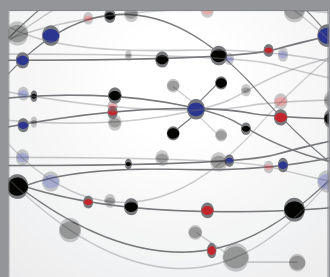

The Scientific World Journal
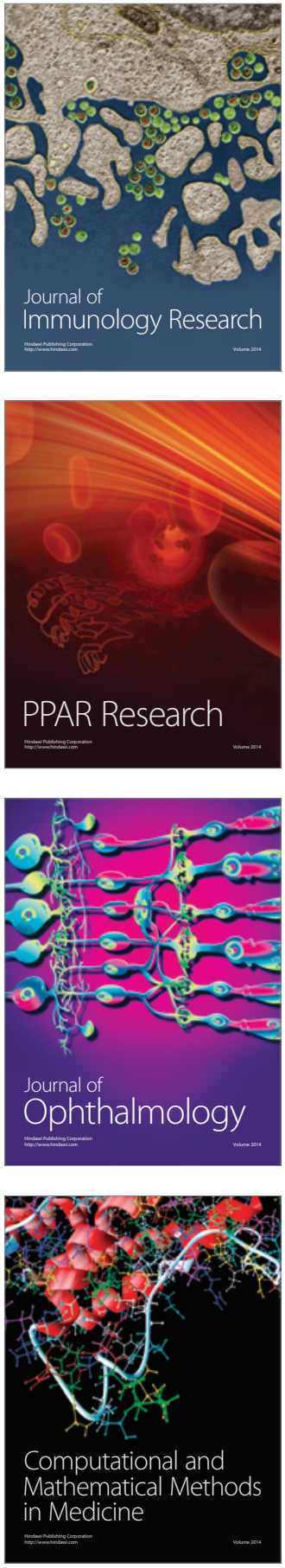

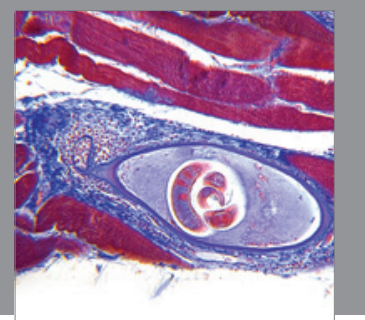

Gastroenterology

Research and Practice
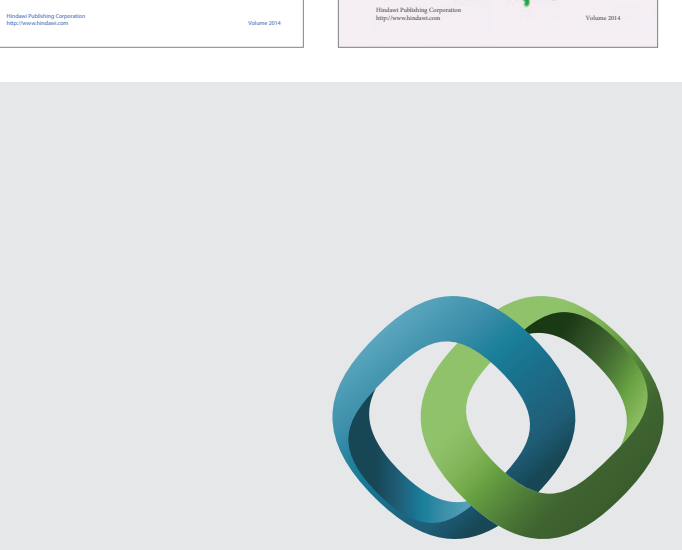

\section{Hindawi}

Submit your manuscripts at

http://www.hindawi.com
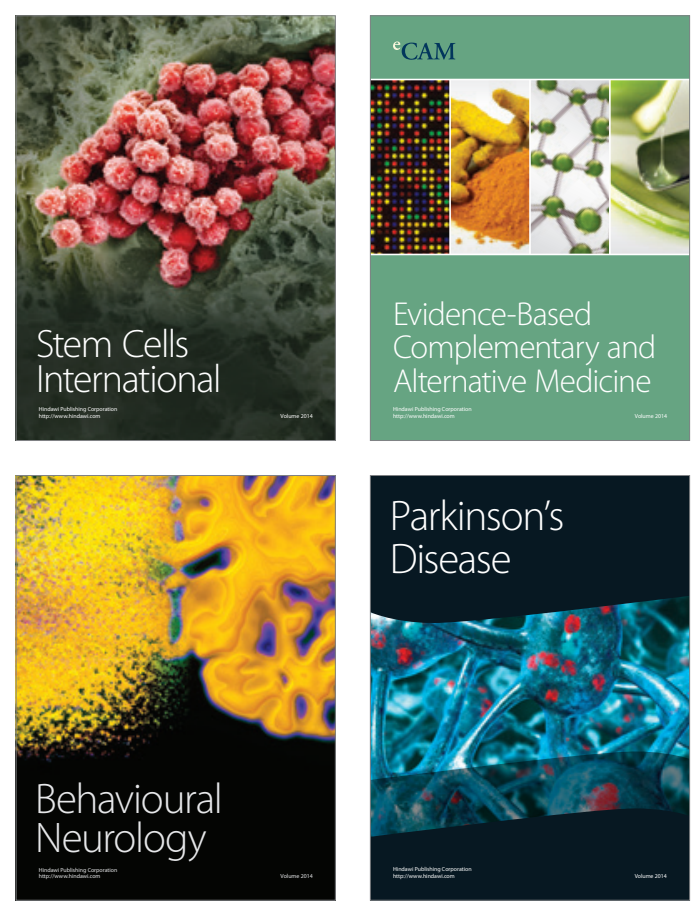

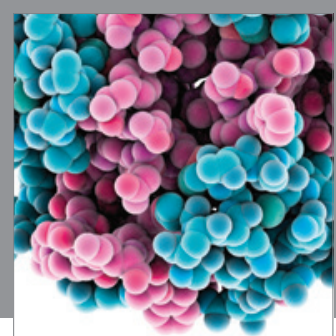

Journal of
Diabetes Research

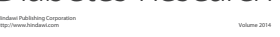

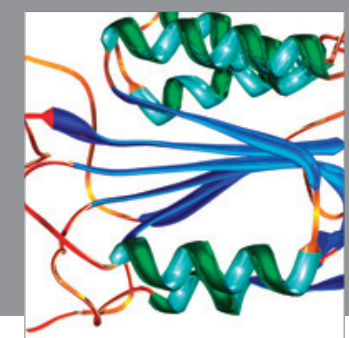

Disease Markers
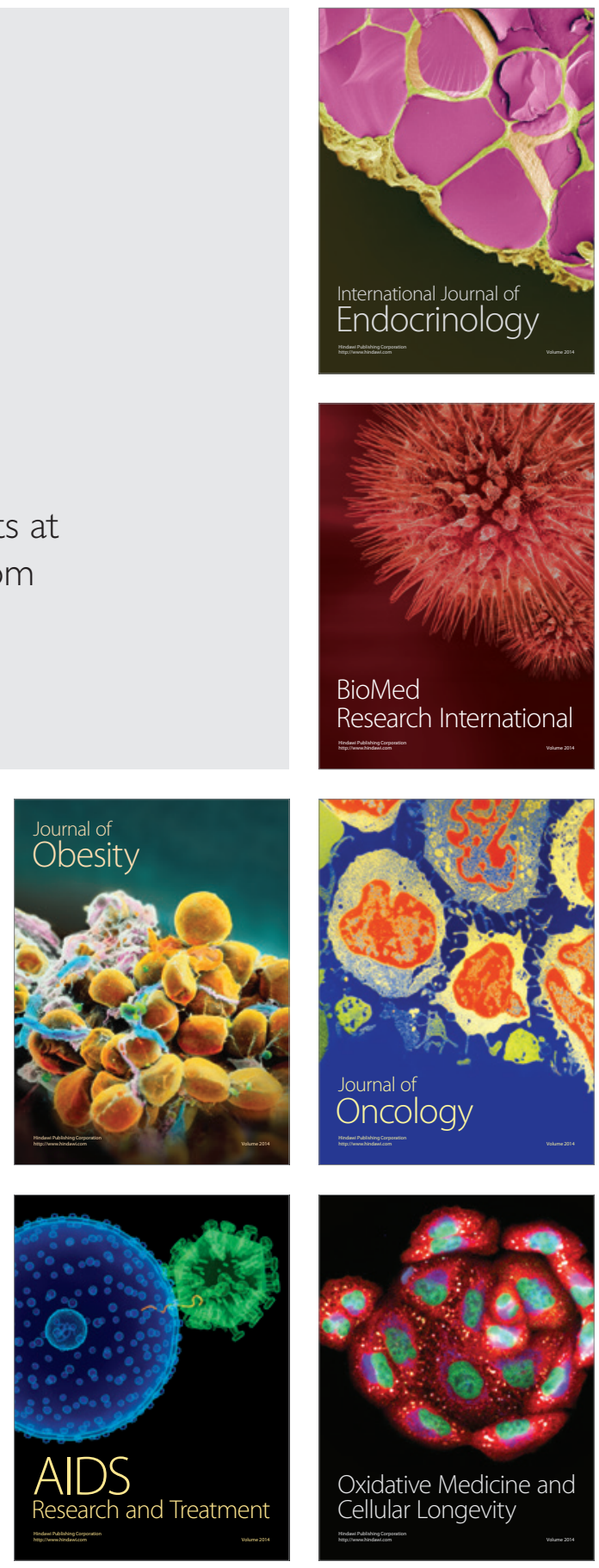